\title{
The Role of the Experimenter in HRI Research - A Case Study Evaluation of Children with Autism Interacting with a Robotic Toy
}

\author{
B. Robins and K. Dautenhahn
}

\begin{abstract}
The general context of the work presented in this paper is assistive robotics with our long-term aim to support children with autism. This paper is part of an investigation into what ways and to what extent a robot can assume the role of a social mediator - encouraging autistic children to interact with the robot, with each other and with co-present adults. The article focuses on the role of the experimenter in these triadic interactions, and provides a case study evaluation of segments of trials where a robot mediated both indirect and direct interactions between children with autism and the experimenter.
\end{abstract}

\section{INTRODUCTION}

Robots, virtual environments and other computer based technologies are more and more being applied in therapy and education. The discussion presented in this paper is part of our investigation in the Aurora project [1] into the potential use of robots as therapeutic or educational 'toys' specifically for use by children with autism, specifically targeting children at the lower end of the autistic spectrum. People with autism have impaired social interaction, social communication and imagination (referred to by many authors as the triad of impairment, e.g. [2]). Our research focuses on ways that robotic systems can engage autistic children in simple interactive activities with the aim of encouraging basic communication and social interaction skills.

The exact causes of autism are still unknown, and at present no cure exists. A variety of therapeutic and educational approaches are known. Any such therapeutic or educational contribution benefits some, but not all children with autism. Our approach to use robots is hoped in future to serve a complementary role: exploiting the fact that children with autism, like most children, show a great affinity towards robots, and using the robot as a useful and programmable toy $[3,4]$, that can be used as a tool in therapeutic and educational context by experience experimenter. This work is complementary to recent research into using robots with children with autism for diagnostic purposes $[5,6]$.

Commonly in Human-Robot Interaction (HRI) research the experimenter is 'invisible' from the point of view of the subjects: he/she not only does not take part in the study but

Ben Robins and Kerstin Dautenhahn are with the Adaptive Systems Research Group, School of Computer Science, University of Hertfordshire, Hatfield, Herts AL10 9AB, U.K (Phone: +44(0)1707281150; Fax: +44 (0)1707 284185; e-mail: \{b.robins,k.dautenhahn\}@herts.ac.uk). is often located out of sight in order not to interfere with and not to provide any bias to the interactions of subjects with the robot. In our approach the contrary applies for the following reasons. Our research investigates how to encourage social interaction skills in children with autism. One of the main impairments of this user group lies in communication and social interaction, therefore the approach taken in the research is that if the children do initiate any interaction with the experimenter they should get a response and encouragement. Thus, the experimenter is not only physically present and 'visible' to the subjects, he/she must include himself as part of the trial, adopting the stance of 'passive participation', to serve as another possible instrument for encouraging social interactions, and to be available and ready to respond to the children should they initiate interaction with him.

\section{A. Autism and Robots technology}

Autism is a lifelong developmental disability that affects the way a person communicates and relates to people around him. People with autism show inability to understand others' intentions, feelings and mental states. They have difficulties in understanding gesture, facial expressions and metaphors and generally have difficulty in forming social relationships and relating to others in meaningful ways. They also have impaired imagination, i.e. the development of play and imaginative activities is limited. Autism is a spectrum disorder and we find a huge variation in abilities and interests among children with autism. For this reason, in our work, interactions of children with autism with robots are evaluated on the level of single children where we find a great variety of types and patterns of interactions characteristic of the children. This approach differs from other HRI research seeking statistical significance in large subject sample sizes.

Robots have been identified as potentially very useful tools to study and possibly enhance the development of social skills in children with autism. To give a few examples: Fasel et al. [7] used simulated and robotic systems to explore the development and dysfunction of shared (joint) attention in toddlers with and without autism. Kozima and Yano, working with a robot that can create and maintain basic joint attention with a human, proposed the development of games that autistic children could play and possibly learn social interaction skills [8]. More recently, Kozima et al developed a small creature-like robot, very simple in appearance, and reported that the robot prompted spontaneous play in children with developmental disorders, and they observe the 
emergence of social communication with the robot and another person [9].

Previous research in the Aurora project illustrated the ability of a mobile robot to provide a focus of attention, and shared attention, in trials with pairs of children with autism [10]. Over the past few years we explored the role of the robot as a social mediator for children with autism using a small humanoid robotic doll. Robins et al. explored robotmediated joint attention in child- adult interactions [11] as well as the role of the robot as a social mediator in childchild interactions [12]. The results documented in [11, 12] are very encouraging and supportive of further research into using robots for therapy of children with autism.

\section{THE ROBOT AND THE TRIALS}

The robot used in the trials is Robota- a humanoid robotic doll (see figure 1). The robot's main body contains the electronic boards and the motors that drive the arms legs and head giving 1 DOF to each. The head and limbs are plastic components of a commercially available doll. Robota has the capability to connect to an array of various sensors, and to support a spectrum of multi modal interactions with children. For a complete description of Robota's hardware see [13].
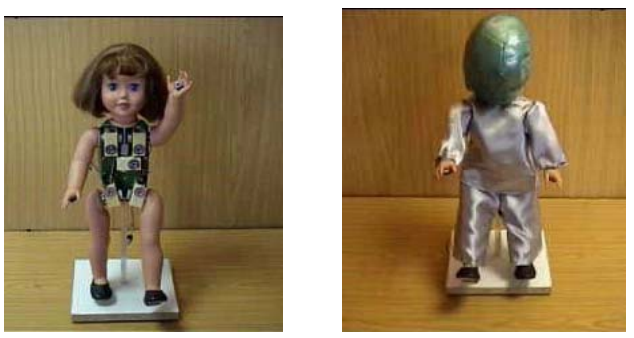

Figure 1: The robot used in the trials. The figure on the left shows the 'undressed' version revealing the robotic parts that control its movement. On the right we see Robota with a face mask and costume (see [14] for a detailed investigation about the robot's appearance).

The robot was programmed to operate in two basic modes:

a) as a 'dancing toy' where it moved its arms, legs and head to the beat of pre-recorded music. We used three types of music - children's rhymes, pop music and classical music, following the teacher's advice as to the children's liking.

b) as a puppet, whereby the experimenter is the puppeteer and, unknown to the children, moves the robot's arms, legs or head by a simple press of buttons on his laptop (Wizardof-Oz approach).

The trials took place in two schools. One is a mainstream primary schools in Essex, UK, with approximately 220 children, which has a special provision unit for 8 children with autism. The second school is a special school for children with moderate learning difficulties, in Hertfordshire, UK. We designed our trials in such a way as to minimize the anxiety and distress the children might find themselves in, caused by a change of routine, being in a novel situation with a new and unusual toy (the robot), and a new person (the investigator). At the same time we wanted to provide a reassuring environment, where the predictability and repetitive behaviour of the robot is a comforting factor. The approach in all the trials has been designed to allow the children to have unconstrained interaction with the robot with a high degree of freedom, and to build a foundation for further possible interactions with peers and adults using the robot as a mediator $[10,12]$

\section{THE ROLE OF THE EXPERIMENTER - A CASE STUDY EVALUATION}

The result of our study showed that with the robot being the focus of joint attention, it mediated interactions between the autistic children and other people - children and adults. Importantly, the trials showed that this role of the robot as a social mediator can be further enhanced when the robot is used as a tool in the hand of an experienced experimenter/operator/therapist. As explained above, the approach adopted in this research is one where the experimenter includes himself as part of the trial, adopting the stance of being available and ready to respond to the children and able to 'seize the opportunity' for any further interactions, should the possibility arise. The experimenter, once he recognised such opportunities, could then secure a triadic mode of interaction between a child, the robot and himself.

This aspect of the crucial role of the experimenter in the trials is demonstrated in the remainder of the paper using case study evaluations. The following are examples where the autistic children used the robot as a mediator and a channel for direct and indirect communication/interaction with the experimenter, cf. [11] for more details on the role of the robot as a focus of joint attention. These examples illustrate the potential role of the robot and of the experimenter in such circumstances where communicative competences in children with autism were elicited.

\section{A. THE CHILDREN}

The children ${ }^{1}$ that participated in these trials are:

- Andy - Age 5, in the reception class. Andy uses only two or three words but is beginning to communicate using the Picture Exchange Communication System (PECS)

- Billy - Age 10, in Year 5. Billy has autism combined with severe learning difficulties. He has

\footnotetext{
${ }^{1}$ All names of children mentioned in this paper are pseudonyms
} 
no verbal language and uses symbols and signs to make choices and express basic needs.

- Jack - Age 7, in year 4. Jack has some limited verbal language which he uses to express needs.

Once a year the schools assess the pupils' performance using the QCA's P-scale method. According to the assessment of their personal and social development level (which was assessed by their teacher six month prior to the trials), in the subject of attention, Andy has been assessed at a level where he pays rigid attention to his own choice of activity, and is highly distractible in activities or tasks led by others. Billy has been assessed at a level where he can attend to an adult directed activity but requires one to one support to maintain his attention. In the area of interacting and working with others, Andy was assessed at a level where he engages in solitary play or work and shows little interest in the activities of those around him. Billy was assessed at a level where he might take part in work/play with one other person and take turns in simple activities with adult support. Jack had joined his school only a short time prior to the start of the trials and no assessments were available.

Three examples of direct and indirect communication / interaction between the children and the experimenter are provided below.

\section{B. CASE STUDY EVALUATION}

i) Example a - The robot became a channel for communication and indirect interaction with the experimenter.

In one of the preliminary trials the child (Jack) engaged in an imitation game with the robot where the robot mirrored the movements of Jack's limbs. Unknown to Jack, the experimenter was operating the robot and responding to Jack's movements as accurately as he could. However, it just happened, on one occasion, that the experimenter unintentionally moved the opposite arm of the robot. Jack giggled and mentioned (to the robot) that this was wrong. After a few turns of correct imitation, the experimenter then introduced, deliberately this time, another mistake in the robot's imitation of Jack's movement - Jack giggled again talking to the robot with affection that this is wrong. The experimenter then introduced more deliberate mistakes, and Jack's laughter and affection directed at the robot grew. Then an important point arrived when Jack realized that the experimenter was operating the robot from his laptop and that it was him who was making the mistakes, so it then became a game between the experimenter and Jack. Whilst Jack still continued to play the imitation game with the robot (Figure 2, image a), after each mistake that the robot made in mirroring Jack's movements (which were deliberately introduced by the experimenter), Jack turned to the experimenter laughing saying 'mistake', 'mistake', this time diverting his affection towards the experimenter (Figure 2, images b \& c). It was very clear at this stage that Jack was actually knowingly playing with the experimenter and sharing his enjoyment with him, whilst standing in front of the robot, initiating movements for the robot to mirror. Thus, Jack was using the robot as a mediator to indirectly interact and play a game with the experimenter. Note, these interpretations (and others proposed in this paper) were confirmed later by therapists/psychologists and teachers in the school when watching the video footage of the trials.

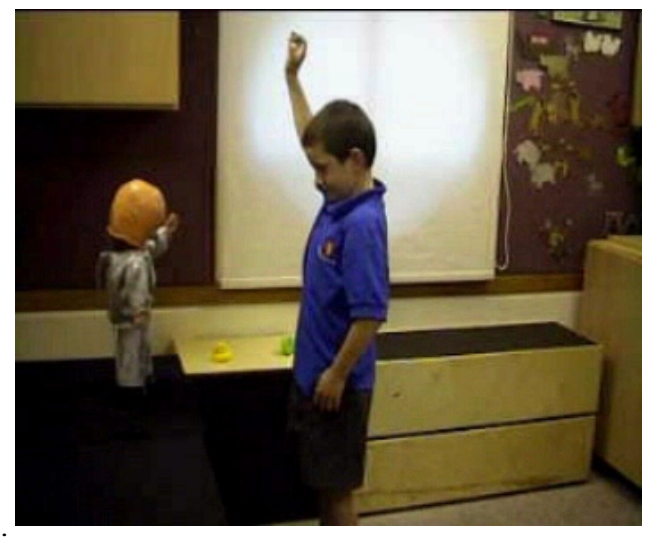

image a

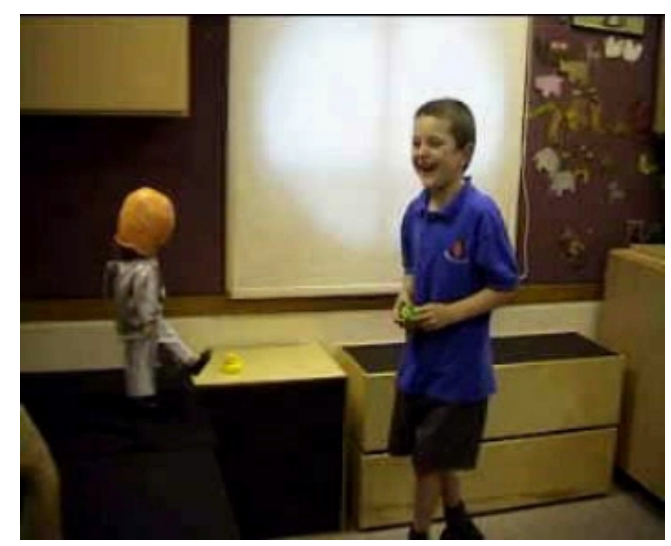

image $b$

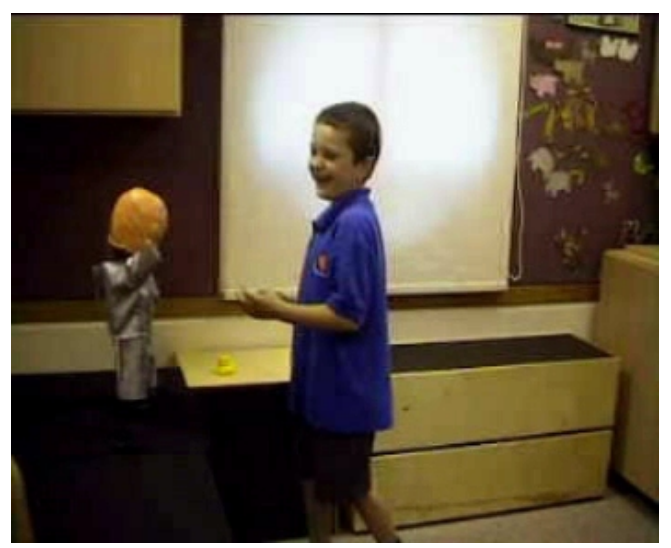

image c

Figure 2: Jack engaged in an imitation game with the robot (image a) and turned to the investigator with giggles each time the robot made a mistake (images b \& c). 
ii) Example $\boldsymbol{b}$ - An unexpected direct communication between the child and the experimenter

This is an example of a child who had participated in many trials before, but had never acknowledged the presence of the experimenter who was sitting next to the robot during the trials. Then, the child, Andy, who has no language skills, unexpectedly came to the experimenter, held his hand and pulled him off his chair to play with him on the floor (fig. 3). Although this was not part of the trial's planned procedure, and was seemingly not a robot related activity, the experimenter supported the child's initiative and decided to participate in this play. After a few mutual giggles whilst kneeling on all fours the experimenter transformed this into a turn-taking game of chasing and retreating (imitation and turn-taking were part of the overall theme of this investigation). They played on the floor for several minutes, after which the experimenter gradually directed the child toward the robot. The robot was operating in its autonomous 'dancing' mode and, while the experimenter was kneeling on one leg in front of it, Andy was sitting on his lap watching the robot moving its head and limbs to the prerecorded music. The experimenter started to teach Andy a simple imitation game by gently moving his head and limbs in response to the robot's movements (fig. 4).

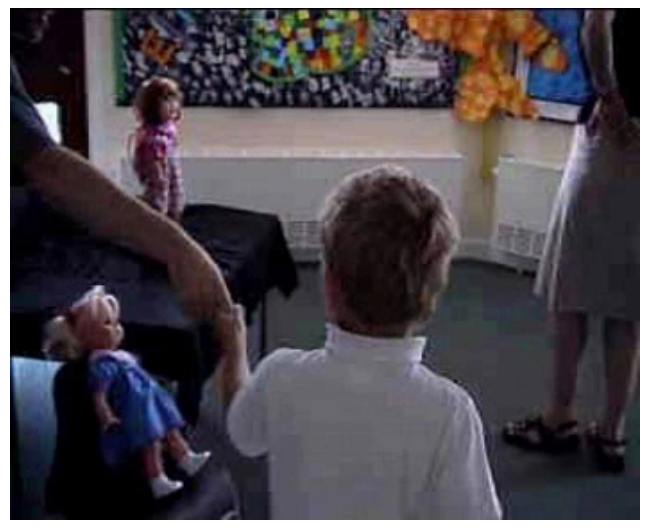

Figure 3: Andy is pulling the experimenter off his chair

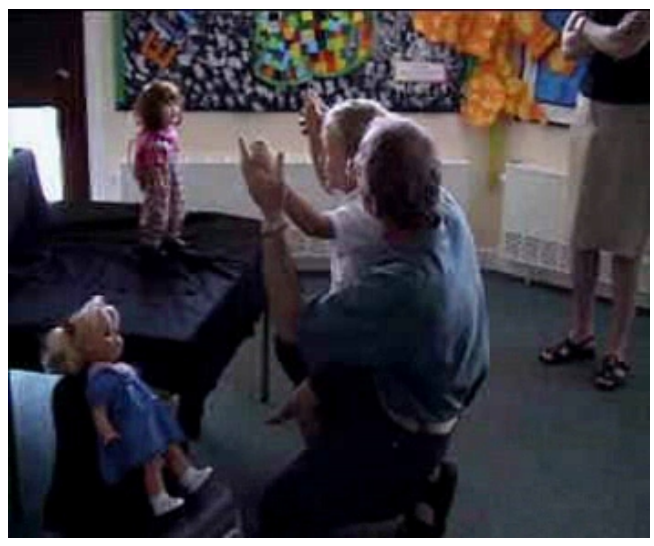

Figure 4: The experimenter is teaching Andy an imitation game.
The autonomous and predictable pattern of the robot's moving arms, legs and head caused Andy to notice a temporarily faulty leg that did not move correctly (fig.5), and he initiated a sequence of non-verbal communication behaviours aimed at conveying this to the experimenter (fig. 6). Detailed analysis of this segment of the trial using conversation analytic evaluation methods can be found in [11].

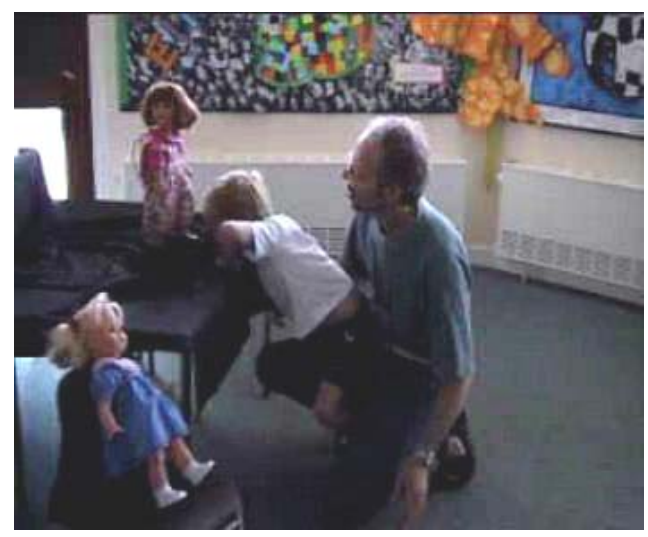

Figure 5: Andy is noticing the temporarily faulty leg of the robot.

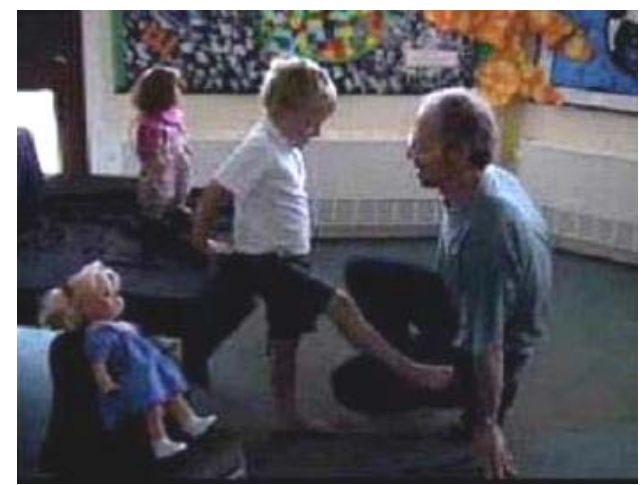

Figure 6: Andy is conveying his discovery to the experimenter.

Clearly, the opportunities for interaction, as described in the above examples, could be used as a tool for intervention, for example if they happened as part of a therapy programme, and could form a platform for subsequently building valued interactions directly between an autistic child and the therapist. While our research does not involve the development of therapy programmes, such results could inform clinical trials that could ultimately lead to new robotassisted therapy programmes.

iii) Example c-An unexpected direct interaction between the child and the experimenter

In figure 7 below, taken from a trial conducted during a longitudinal study [4], is a still shot taken out of a sequence where for the first time, the child (Billy) acknowledged the presence of the investigator. Although Billy has participated in several trials before, up to this point he only interacted 
with the robot, and completely ignored the investigator. In this trial he surprised everyone and came, in a very joyful way, to sit on the investigator's lap for a few moments, giggling most of the time. The investigator took this opportunity to direct the boy's attention to the robot (fig. 7). Billy, responding to the investigator's gaze and pointing, stood up and moved towards the robot whilst continuing to hold the investigator's hand (fig. 8 below).

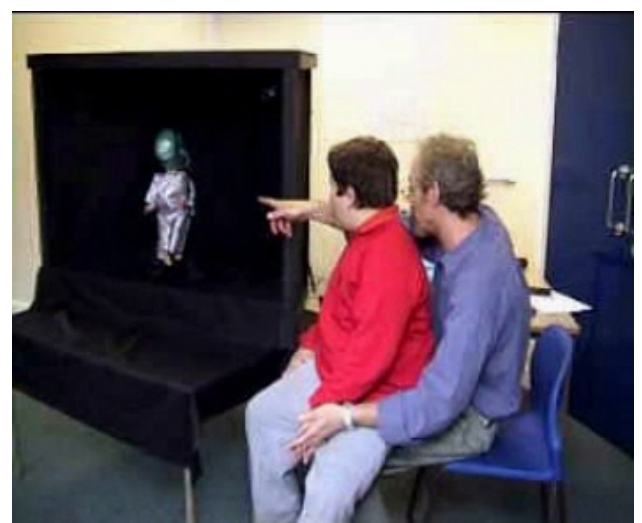

Figure 7: Billy is sitting on the experimenter's lap following his gaze to the direction of the experimenter's pointing

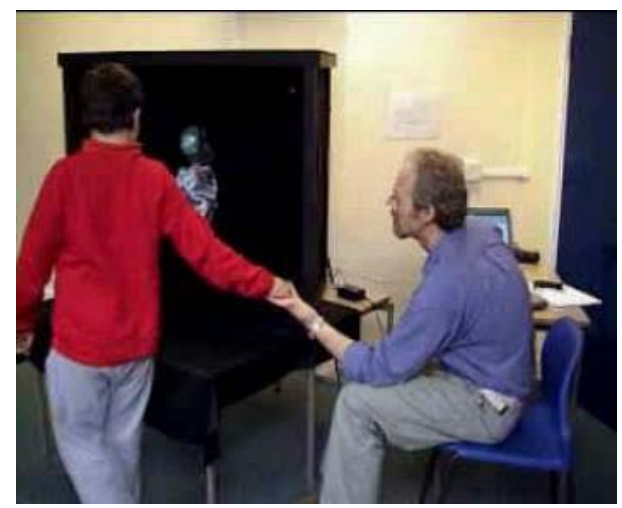

Figure 8: Billy is moving toward the robot whilst continuing to hold the investigator's hand

We believe that this sharing of experiences is an important aspect of the work, since the human contact gives significance and (emotional, intersubjective) meaning to the experiences with the robot that are otherwise mechanical.

\section{CONCLUSION \& LESSONS LEARNT}

Since one of the main impairments of our user group lies in communication and social interaction, our research takes the approach that if the children do initiate any interaction with the experimenter they should get a response and encouragement. Thus the experimenter should include himself as part of the trial, adopting the stance of 'passive participation', to be another possible instrument for encouraging social interactions, available and ready to respond to the children should they initiate interaction with him. This role of the experimenter is very different from the role usually adopted in HRI trials, inspired e.g. by work in experimental psychology where a great emphasis lies in avoiding any experimenter bias and interference with the trials. In our work, participation of the experimenter in the trials is not only acceptable, but necessary in order to meet the social and therapeutic needs of children with autism.

Furthermore, similar to a therapist in a therapy session, the experimenter should be 'in contact' with the children all the time with the 'finger on the pulse' to be able to respond accurately to the children (via the robot when they interact with the robot) and to 'seize the opportunity' for further possible interactions should they arise even if it means the need to change the pre-planned procedure of the trial. Although working according to a plan, the experimenter needs to be able to deviate from it and grasp any opportunity to expand and develop the interactions. In such situations an experienced experimenter can respond from 'gut feelings' or 'intuition' but also needs to concentrate hard and think quickly in selecting the most valuable variation on the basic theme of the original trial plan. At the same time this process requires a great deal of awareness in order to maintain the overall containment and structure of the trial.

To conduct successful and safe research using this approach requires an experimenter with a lot of experience in therapy and with access to expert advice (in the field of autism). This points out the crucial necessity for interdisciplinary approaches specifically when using robots in autism therapy, but which could also generalize to other contexts where robots are used in therapeutic contexts.

To conclude, this paper highlighted a specific approach in an application area of HRI where the involvement of the experimenter is crucial for the success of the trials. Such work emphasises the multi-disciplinary nature of HRI studies, where specific application areas require specific approaches, methods and methodologies that need to be informed by other, non-robotics related, disciplines.

\section{ACKNOWLEDGMENT}

We are grateful to the teaching staff, parents and children at Bentfield Primary School and at Middleton School. Special thanks go to the headteacher at Bentfield school, Mr Draper, and to the head of autism provision at Middleton School, Mrs. Philp for their continued support. We would like to thank Aude Billard at EPFL for her supportive collaboration in the context of using the humanoid robot developed by her in our trials.

\section{REFERENCES}

[1] AURORA, URL: http://www.aurora-project.com/last accessed 06/03/06, 2006.

[2] L. Wing, The Autistic Spectrum. London: Constable Press, 1996. 
[3] K. Dautenhahn and I. Werry, "Towards interactive robots in autism therapy: Background, motivation and challenges," Pragmatic and Cognition, vol. 12,pp. 1-35, 2004.

[4] B. Robins, K. Dautenhahn, R. te-Boekhorst, and A. Billard, "Robotic assistants in therapy and education of children with autism: can a small humanoid robot help encourage social interaction skills?," Universal Access in the Information Society, vol. 4:2, 2005.

[5] B. Scassellati, "How social robots will help us to diagnose, treat, and understand autism. 2005," Proc. 12 th International Symposium of Robotics Research (ISRR). San Francisco, CA. Oct., 2005.

[6] B. Scassellati, "Quantitative metrics of social response for autism diagnosis," Proc. 14th International Workshopon Robot and Human Interactive Communication (ROMAN05), Nashville, TN, 2005.

[7] I. Fasel, D. Gedeon, J. Triesch, and J. Movellan, "Combining Embodied Models and Empirical research for Understanding the Development of Shared Attention," Proc. The 2nd International Conference on Development and Learning (ICDL'02), 2002.

[8] H. Kozima and H. Yano, "Designing a robot for contingencydetection game," Proc. Workshop on Robotic and Virtual Interactive Systems in Autism Therapy, 2001.

[9] H. Kozima, Nakagawa, C., Yasuda, Y., "Designing and observing human-robot interactions for the study of social development and its disorders," Proc. The 6th IEEE International Symposium on Computational Intelligence in Robotics and Automation - CIRA 2005, 2005.

[10] I. Werry, K. Dautenhahn, B. Ogden, and W. Harwin, "Can Social Interaction Skills Be Taught by a Social Agent? The Role of a Robotic Mediator in Autism Therapy," in Proc. CT2001, The Fourth International Conference on Cognitive Technology: Instruments of Mind, LNAI 2117, M. Beynon, C. L. Nehaniv, and K. Dautenhahn, Eds. Berlin Heidelberg: Springer-Verlag, 2001, pp. 57-74.

[11] B. Robins, P. Dickerson, P. Stribling, and K. Dautenhahn, "Robotmediated joint attention in children with autism: A case study in a robot-human interaction," Interaction studies: Social Behaviour and Communication in Biological and Artificial Systems, vol. 5:2 John Benjamins Publishing Company, Amsterdam,pp. 161-198, 2004.

[12] B. Robins, P. Dickerson, P. Stribling, and K. Dautenhahn, "Robots as embodied beings - Interactionally sensitive body movements in interactions among autistic children and a robot," Proc. 14th IEEE International Workshop on Robot and Human Interactive Communication -RO-MAN'05, 2005.

[13] A. Billard, "Robota: Clever Toy and Educational Tool," Robotics and Autonomous Systems,pp. (42):259-269, 2003.

[14] B. Robins, K. Dautenhahn, R. t. Boekhorst, and A. Billard, "Robots as Assistive Technology - Does Appearance Matter?," Proc. 13th IEEE International Workshop on Robot and Human Interactive Communication - RO-MAN , Kurashiki, Japan, 20-22 September, 2004. 